\title{
Radiation tolerance enhancement of silicon photonics for HEP applications
}

\author{
Andrea Kraxner*, Stephane Detraz, Lauri Olantera, Carmelo Scarcella, Christophe \\ Sigaud, Csaba Soos, Carmine Stile, Jan Troska and Francois Vasey \\ Experimental Physics Department, CERN, CH-1211 Geneva, Switzerland \\ E-mail: andrea.kraxner@cern.ch
}

\begin{abstract}
Silicon photonics modulators and photodiodes are being investigated for use in optical links for High Energy Physics experiments. In order to withstand the harsh environment in the innermost detector regions of the Large Hadron Collider at CERN and in future High Energy Physics experiments beyond the Large Hadron Collider, components will have to be resistant against extreme levels of radiation. First, we show that Mach-Zehnder modulators, which lost their functionality after X-ray irradiation, can be fully recovered by applying a forward bias after irradiation. Devices irradiated and recovered withstand the same TID when re-irradiated. Furthermore, it is presented that by applying a forward bias already during irradiation, the irradiation-induced degradation can be compensated. The possibility of device recovery could lead to a tremendous increase of radiation resistance of the optical links. Additionally, the resistance against displacement damage and ionizing radiation of silicon germanium photodiodes is presented.
\end{abstract}

Topical Workshop on Electronics for Particle Physics (TWEPP2018)

17-21 September 2018

Antwerp, Belgium

${ }^{*}$ Speaker. 


\section{Introduction}

Optical links have become vital components in High Energy Physics Experiments (HEP) at CERN to transport data generated from the particle detectors and other control points along the Large Hadron Collider (LHC) in hostile radiation environments to the processing electronics. In order to withstand ever-higher radiation levels and to handle the increasing data volumes, alternatives to laser diode-based transmitters [1] have to be investigated.

In this work silicon photonics devices are investigated and their applicability for use in optical links in future HEP experiments is discussed. The main building blocks, modulators and photodiodes, are tested towards their radiation hardness. Previous work has shown that silicon photonics Mach-Zehnder modulators (MZMs) are relatively insensitive to neutron radiation, but show strong degradation with ionizing X-ray radiation. It was further presented, that with customized MZM designs, increasing the rib height of MZMs (shallow-etch device), the resistance against X-ray radiation can be improved $[2,3,4,5,6]$. Recently, the influence of forward biasing on the radiation hardness was reported and a strong post-irradiation annealing effect was demonstrated [7].

Since then a deeper investigation of this injection enhanced annealing behaviour after and during X-ray irradiation was carried out. In this work we will show that a full recovery of the silicon photonics MZMs is possible by applying a forward bias for a long enough time period. Furthermore, we will show measurement data indicating that by frequently applying a sufficiently high forward current during the irradiation the radiation induced degradation of the device performance can be compensated.

Additionally, to prove the radiation hardness of an optical link based on silicon photonics neutron and X-ray irradiation results of SiGe photodiodes, produced on the same silicon photonics platform as the MZMs, are presented.

\section{Forward bias annealing}

In [7] a strong post-irradiation annealing effect by applying a forward bias to a device that was irradiated with a $10 \mathrm{keV} \mathrm{X}$-ray source up to a point where it was not functional anymore was presented. It was shown that after applying a forward bias for a sufficient amount of time the preirradiation performance can be restored. To test the efficiency of the annealing and to proof that full recovery is achieved, re-irradiation tests were performed. Deep etch as well as shallow etch devices were first irradiated up to device failure and subsequently annealed by applying a forward current of $10 \mathrm{~mA}$ for 24 hours followed by 24 hours of $-3 \mathrm{~V}$ reverse bias. The reverse bias step was introduced as this is equivalent with the MZM operation condition to make sure that there is no reverse annealing effect. After it was ensured that the device performance was back to the preirradiation state the devices were re-irradiated using the same conditions as in the first irradiation run. Both irradiation runs were performed at $25^{\circ} \mathrm{C}$ with a dose rate of $14 \mathrm{~Gy} \mathrm{~s}^{-1}$ and up to a TID of $3 \mathrm{MGy}$. In Figure 1 the relative phase shift at $-1 \mathrm{~V},-2 \mathrm{~V}$ and $-3 \mathrm{~V}$ reverse bias for the first irradiation run (in red) and the second irradiation run (in blue) are shown for (a) a deep-etch device and (b) a shallow-etch device. It can be seen that for both designs the radiation resistance is the same in both irradiation runs. Even a slight improvement in the second run was observed. This can indicate defects being present in the device already before the first irradiation which are removed 
by the annealing. The fact that the device could withstand the same TID after annealing proofs a full recovery.

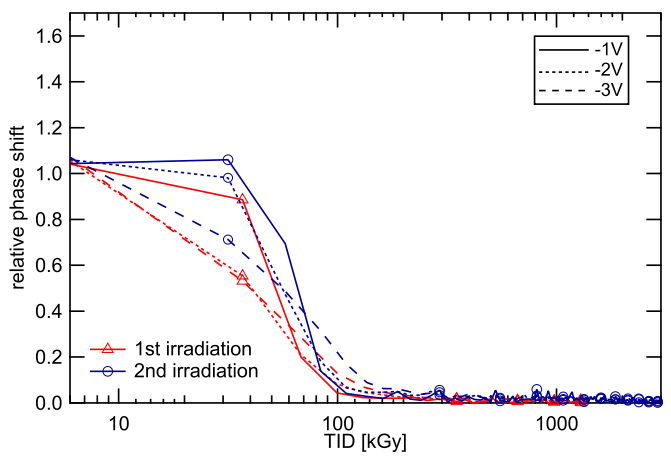

(a) deep-etch

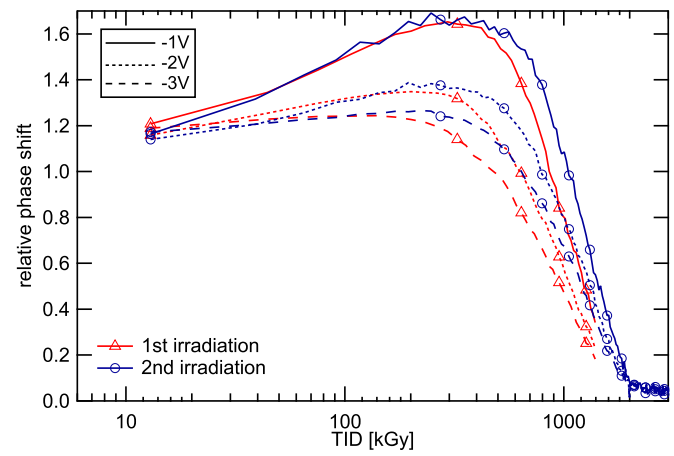

(b) shallow-etch

Figure 1: Relative phase shift at $-1 \mathrm{~V},-2 \mathrm{~V}$ and $-3 \mathrm{~V}$ of the first irradiation and second irradiation after forward bias annealing of the same (a) deep-etch and (b) shallow-etch devices.

The next question was if it is possible to compensate for the radiation effects already during the irradiation using the same mechanism. It was shown that applying $2 \mathrm{~mA}$ for 1 minute in every measurement cycle (every 30 minutes) did lead to a recovery post-irradiation but had only a very small positive effect on the radiation resistance during the irradiation. In post-irradiation experiments we have observed that either a longer time of forward current or a higher forward current increases the effect of recovery. Using this knowledge samples were again irradiated up to a TID of 3 MGy using a higher forward bias. In Figure 2a the relative phase shift for a deep-etch device and in Figure $2 \mathrm{~b}$ for a shallow-etch device is plotted where $10 \mathrm{~mA}$ were applied for 1 minute in every measurement cycle (blue) compared to a device where no forward bias at all was applied (red). In the case of the deep etch as well as the shallow etch device the phase shift did not degrade at all with increasing TID proofing the assumption that with an high enough current the radiation induced degradation can be compensated already during the irradiation.

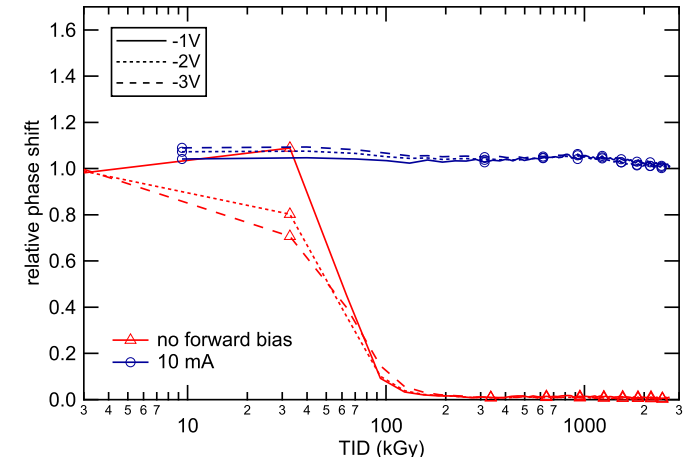

(a) deep-etch

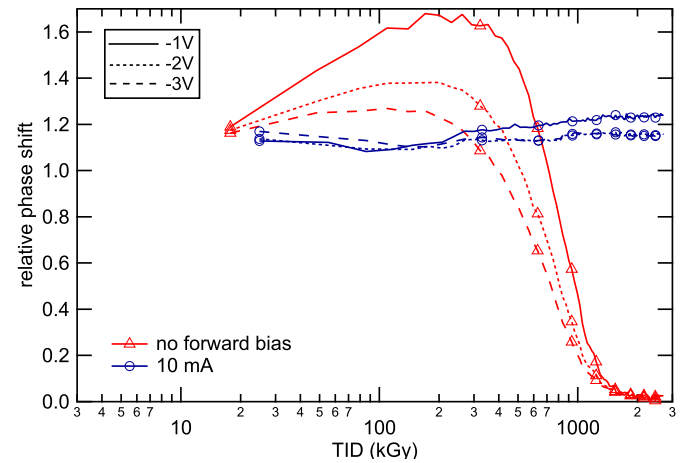

(b) shallow-etch

Figure 2: Relative phase shift at $-1 \mathrm{~V},-2 \mathrm{~V}$ and $-3 \mathrm{~V}$ with no forward bias applied (red) and $10 \mathrm{~mA}$ forward bias applied for 1 minute every measurement cycle (blue) for (a) a deep-etch and (b) a shallow-etch device. 


\section{Irradiation resistance of $\mathrm{SiGe}$ Photodiodes}

In order to ensure the radiation resistance of the whole system forming an optical link also photodiodes have to be tested. In standard silicon photonics platforms silicon germanium ( $\mathrm{SiGe})$ photodiodes with germanium as absorbing material are used. We present the results of X-ray tests (Figure 3) as well as neutron tests (Figure 4) performed on two different SiGe photodiode types. The photo current and the dark current were measured in-situ during the irradiation tests. In Figure 3 and Figure 4 it can be seen that there is no significant change due to ionizing radiation as well as due to displacement damage. The decrease in responsivity of PD2 in Figure 4 starting during irradiation and continuing during the recovery time with a sudden recovery at the end can be explained by the strong polarization dependence of the grating couplers used to couple the light from the fibers into the chip and a change of input polarization due to a moving fiber.
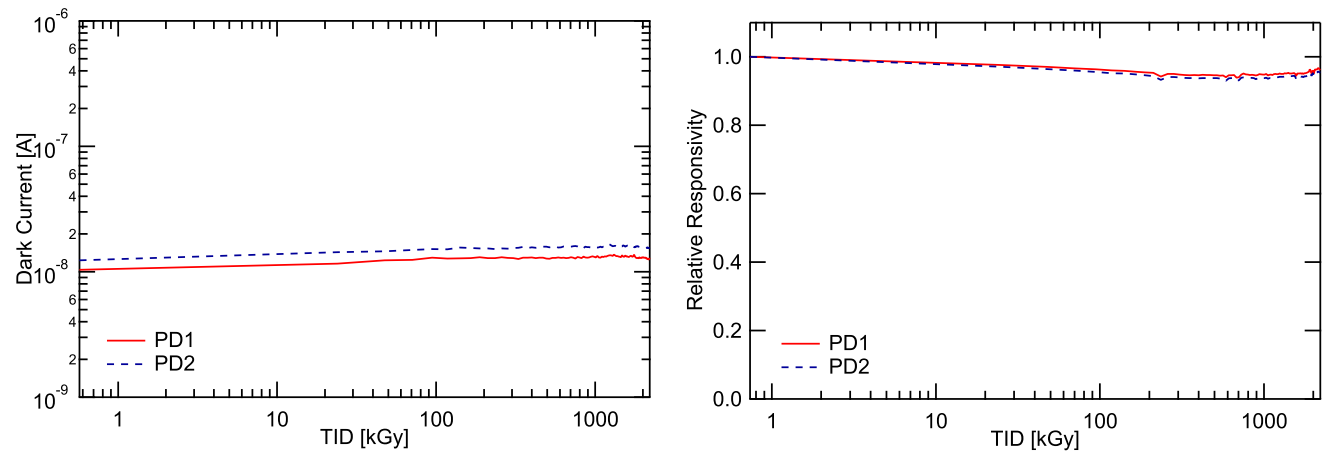

Figure 3: Dark current and relative responsivity at $-1 \mathrm{~V}$ reverse bias of two photodiode types during $\mathrm{X}$-ray irradiation.

\section{Conclusion}

It was proven that by applying a forward bias MZMs can be fully recovered regaining the same resistance against ionizing radiation as before the first irradiation. Furthermore, it was shown that by applying a sufficiently high forward bias the radiation effects can be compensated and no device degradation was observed during an irradiation up to $3 \mathrm{MGy}$. This recovery process increases the radiation resistance of the devices tremendously and a forward bias could be applied in phases where the operation of the HEP experiments is paused eg. during shut-downs, technical stops or interfills. The shown compensation mechanism can be applied to the standard optimized devices by foundries. This eliminates the need for custom radiation hard designs (shallow etch) which come with a drawback in performance. Additionally, the radiation hardness of SiGe photodiodes was presented. Up to the tested TID and fluence no significant degradation due to displacement damage or TID was observed.

\section{References}

[1] J. Troska, S. Detraz, S. S. E. Nasr-storey, P. Stejskal, C. Sigaud and C. Soos, Radiation Damage Studies of Lasers and Photodiodes for Use in Multi-Gb /s Optical Data Links, IEEE Transactions on Nuclear Science 58 (2011) 3103-3110. 

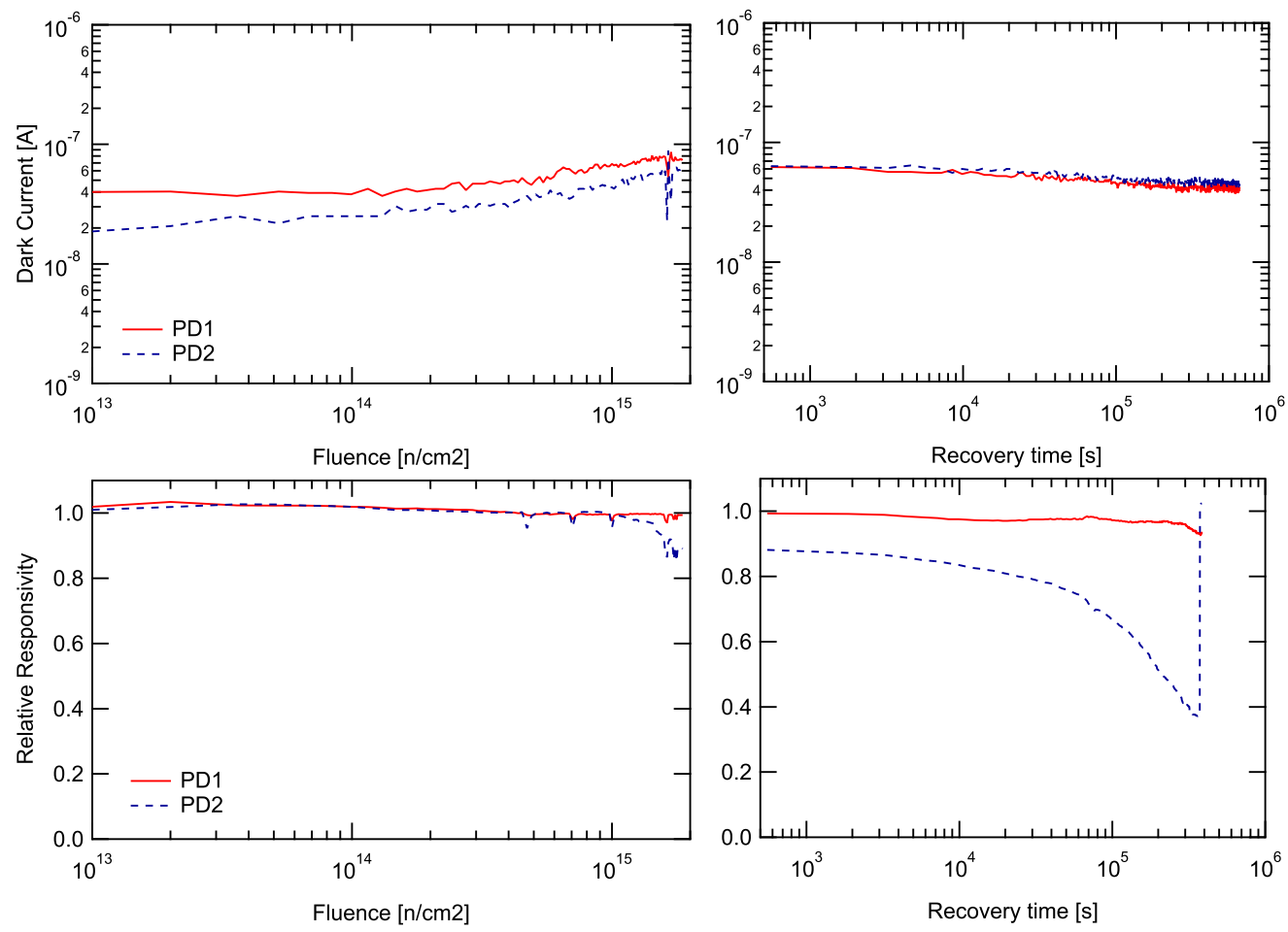

Figure 4: Dark current and relative responsivity at $-1.5 \mathrm{~V}$ reverse bias of two photodiode types during neutron irradiation and recovery time post-irradiation.

[2] M. Zeiler, S. S. El Nasr-Storey, S. Detraz, A. Kraxner, L. Olantera, C. Scarcella et al., Radiation Damage in Silicon Photonic Mach-Zehnder Modulators and Photodiodes, IEEE Transactions on Nuclear Science 64 (nov, 2017) 2794-2801.

[3] S. Seif El Nasr-Storey, F. Boeuf, C. Baudot, S. Detraz, J. M. Fedeli, D. Marris-Morini et al., Silicon Photonics for High Energy Physics Data Transmission Applications, IEEE 11th International Conference on Group IV Photonics (GFP) (2014) 1-2.

[4] S. E. Nasr-Storey, S. Detraz, L. Olantera, C. Sigaud, C. Soos, G. Pezzullo et al., Neutron and X-ray irradiation of silicon based Mach-Zehnder modulators, Journal of Instrumentation 10 (mar, 2015) C03040-C03040.

[5] M. Zeiler, S. Detraz, L. Olantera, G. Pezzullo, S. Seif El Nasr-Storey, C. Sigaud et al., Design of Si-Photonic structures to evaluate their radiation hardness dependence on design parameters, Journal of Instrumentation 11 (2016) .

[6] M. Zeiler, S. Detraz, L. Olantera, S. S. E. Nasr-storey, C. Sigaud, C. Soos et al., Radiation hardness evaluation and phase shift enhancement through ionizing radiation in silicon Mach-Zehnder modulators, in Radiation Effects on Components and Systems (RADECS), (Bremen, Germany), 2016.

[7] A. Kraxner, S. Detraz, L. Olantera, C. Scarcella, C. Sigaud, C. Soos et al., Investigation of the Influence of Temperature and Annealing on the Radiation Hardness of Silicon Mach-Zehnder Modulators, IEEE Transactions on Nuclear Science 65 (aug, 2018) 1624-1631. 